\title{
Far-field control of nanoscale hotspots by near-field interference
}

\author{
Anshuman Singh, ${ }^{\dagger,+}$ James T. Hugall, ${ }^{\dagger}$ Gaëtan Calbris, ${ }^{\dagger}$ and Niek F. van Hulst ${ }^{*},+, \mathbb{l}$ \\ $\dagger I C F O-I n s t i t u t$ de Ciencies Fotoniques, The Barcelona Institute of Science and Technology, 08860 \\ Castelldefels (Barcelona), Spain \\ $\ddagger$ Raytheon BBN Technologies, Cambridge, Massachusetts 02138, USA \\ IICREA- Instutució Catalana de Recerca i Estudis Avançats, 08010 Barcelona, Spain \\ E-mail: niek.vanhulst@icfo.eu
}

\begin{abstract}
Optical nanoantennas coherently scatter incident optical radiation, creating a localized electromagnetic near-field imprinted with sub-wavelength phase distribution, rapidly changing in space. The nanostructuring of phase by a nanoantenna makes the scattered field locally interfere with the incident far-field, whose phase lacks nanostructuring and depends only on wave propagation in time and space. Depending on the resonance conditions and material properties, the interference strongly modifies the resultant near-field intensity of the nanoantenna. Here, we present direct imaging of the local near-field interference effect in the near-field of a resonant dipole nanoantenna, supported by numerical calculations. We use single fluorescent emitters as local detectors to map the field distribution of nanoantennas with nanometer precision in the presence of an incident field. Taking advantage of the dipole orientation of the molecule we probe the vectorial character of the near-field interference map. Exploiting the local interference we demonstrate position control of the localized field, i.e. the plasmonic hotspots in the nearfield of a nanoantenna. The results shown in this paper are important for applications where the enhancement and precise hotspot position at the nanoantenna are crucial, such as in high
\end{abstract}


resolution fluorescence imaging. Moreover, the interference effect can be exploited to shape the nanoantenna near-field to produce a tailored optical response such as polarization-controlled nanoscale hotspot switching, as we show here.

Keywords: local interference, near-field phase, polarization switching, nanoantenna, vectorial field, plasmonics

\section{Introduction}

Intense electromagnetic hotspots can be created using plasmonic nanostructures, such as optical nanoantennas, ${ }^{1,2}$ which confine light close to metal-insulator interfaces by coupling it to localized plasmons inside the metal surface. ${ }^{3,4}$ This leads to localized intensity hotspots orders of magnitude more intense than the illuminating field. Such field localization is useful to enhance the interaction of light and matter down to the single molecule level, even in especially weak processes, such as surface-enhanced Raman scattering (SERS), where enhancements of signal up to $10^{14}$ times have been observed. ${ }^{5}$ Inside these nanosized, sub-diffraction limited volumes, ${ }^{6-8}$ the highly localised electromagnetic field takes on a 3D vectorial near-field nature with both amplitude and phase rapidly changing on the nanometer scale. ${ }^{9-13}$ Such local phase patterns are being used to engineer optical meta-surfaces. ${ }^{12,14,15}$

Since the induced or scattered plasmonic electric field $\mathbf{E}_{s}$ is many times stronger than the excitation field $\mathbf{E}_{i n}$ at the centre of the hotspots, any effect of interference between the coherent induced scattering and the excitation field are negligible as the induced field dominates and therefore such interferences are rarely mentioned in the literature. The induced field, however, rapidly decays away from the hotspot centre, and moving slightly away from this centre leads to areas where the amplitude of the induced field is comparable to that of the excitation field. The induced field at these positions remains deeply sub-wavelength with strong vectorial structure, just like at the centre of the hotspot, but here interference with the excitation field becomes a significant effect and the overall resultant field now depends on properties such as the polarization, angle of incidence of the 
excitation, and crucially on the phase difference of the scattered field relative to the excitation field.

This means that the nanosized field structure can be shaped and changed not only by changing the plasmonic nanostructure itself but equally by altering the nature of the excitation field. Interference effects can thus be used to dynamically tailor and control the local field of a nanostructure with simple changes to the illumination condition. This is of interest to applications in nanooptical circuitry ${ }^{16-18}$ where switching and manipulation of nanosized hotspots can occur without the need to physically manipulate the nanostructure itself.

In this paper, we present the direct experimental observation of the near-field or local interference effect, supported by numerical calculations. Our detailed study of the local interference in the near-field of a resonant metallic nanoantenna, provides full insight into the underlying mechanisms responsible for this interference, and moreover how manipulate it. We use single fluorescent dye molecules as local nanosized probes to directly probe the local interference pattern around an optical nanoantenna. We show the effect of the vectorial field distribution of the nanoantenna on the interference by exploiting the specific dipole orientation of each single molecule in space. The right combination of the incident polarization and nannoantenna design can be applied to symmetrically modify the near-field distribution and control the position of a hotspot at the nanoantenna, showing potential for optical switching in nanoscale all optical circuitry.

\section{Local interference from a nanoantenna}

When illuminated by an incoming propagating wave at frequency $\omega_{0}$, a nanostructure elastically scatters the incoming field. The total field $\mathbf{E}_{t o t}(\mathbf{r})$ at any $\mathbf{r}$ around the nanostructure is a superposition of both the incident field $\mathbf{E}_{i n}(\mathbf{r})$, and the scattered field $\mathbf{E}_{s}(\mathbf{r})$. The phenomena of the local interference can be understood by considering the near-field of the nanostructure i.e. the nonpropagating part of the scattered field. The near-field is generally characterized by a subwavelength confinement possessing a 3D vectorial distribution. The vectorial field can be decomposed along the three Cartesian axes $(\mathrm{x}, \mathrm{y}, \mathrm{z})$. The total intensity at position $\mathbf{r}$ is the time average of $\mathbf{E}_{t o t}(\mathbf{r})$ and 
is given by $I_{\text {tot }}(\mathbf{r})=\left|\mathbf{E}_{\text {tot }}(\mathbf{r})\right|^{2}$. Therefore, $I_{t o t}(\mathbf{r})$ can be expressed as:

$$
I_{t o t}(\mathbf{r})=\left|\mathbf{E}_{i n}\left(\mathbf{r}, \omega_{0}\right)\right|^{2}+\left|\mathbf{E}_{s}\left(\mathbf{r}, \omega_{0}\right)\right|^{2}+2 \sum_{i}\left|E_{i n}^{i}\left(\mathbf{r}, \omega_{0}\right)\right|\left|E_{s}^{i}\left(\mathbf{r}, \omega_{0}\right)\right| \cos \left(\Delta \varphi_{i}\left(\mathbf{r}, \omega_{0}\right)\right)
$$

where $i=\mathrm{x}, \mathrm{y}$, or $\mathrm{z}$ and $\Delta \varphi$ is the phase difference between the $i^{t h}$ component of $\mathbf{E}_{\text {in }}$ and $\mathbf{E}_{s}$. The last term of Eq. 1 represents the contribution from the interference of the incoming and scattered fields for each spatial direction. Each component of the vectorial field exhibits a spatial structure with a strong variation in amplitude and phase. ${ }^{13,19,20}$ Based on the amplitude and phase relations with the incoming field components, the resulting intensity can be strongly modified, thus presenting a sub-wavelength interference pattern.

To illustrate this effect, we consider the interference field pattern around a metallic nanorod

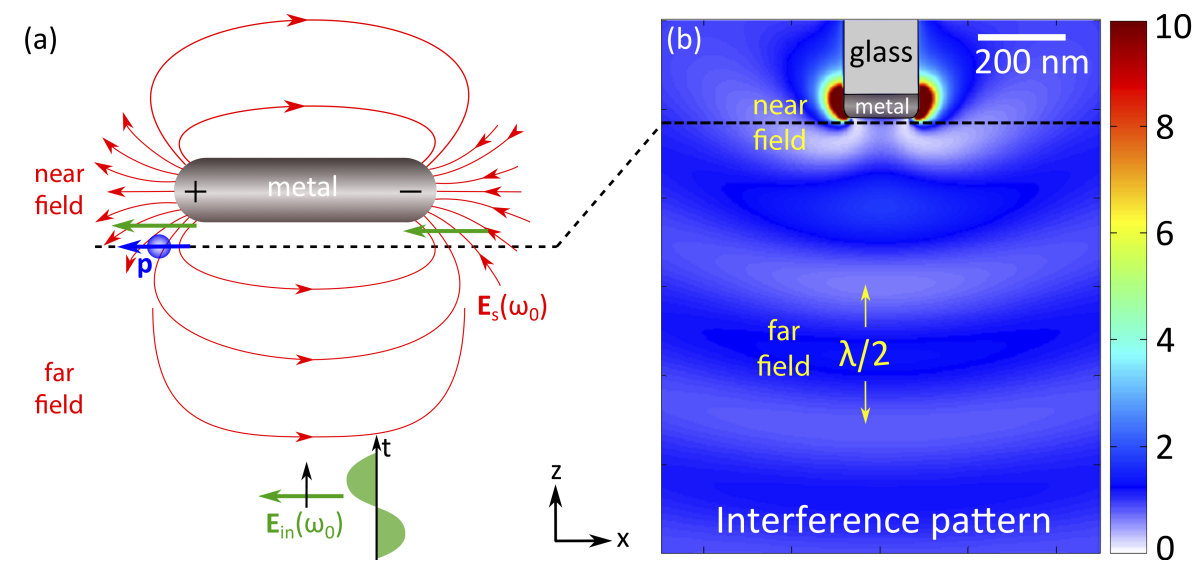

Figure 1: Local near-field interference at a nanoantenna. (a) Schematic of a dipole nanoantenna and its scattered field lines $\mathbf{E}_{s}$ (red) excited by a linearly polarized propagating incident field $\mathbf{E}_{i n}$ (green) both at the same frequency $\omega_{0}$. The figure represents a snap shot of the charge distribution and resulting field at a fixed time in an optical cycle. Since the nanoantenna elastically scatters the impinging radiation, the two fields interfere. A molecule (blue) with dipole moment $\mathbf{p}$ acts as a local field detector and sees the superposition of $\mathbf{E}_{s}$ and $\mathbf{E}_{i n}$ as a function of its position and orientation. (b) The calculated X-field intensity $I_{\text {tot }}^{x}(\mathbf{r})$ pattern of the nanoantenna (metal) in the xz-plane shows an interference fringe pattern with period $\lambda / 2$ in the far-field. Here the aluminum nanoantenna is shown suspended on a glass fiber to match later experimental conditions and has dimensions: length $(\mathrm{x})=170 \mathrm{~nm}$, width $(\mathrm{y})=50 \mathrm{~nm}$ and, height $(\mathrm{z})=50 \mathrm{~nm}$, and is excited with linearly polarized light resonant along its long axis (x) at $\lambda=633 \mathrm{~nm}$. The dashed line in (a) and (b) is the plane $15 \mathrm{~nm}$ below the nanoantenna, where we place the probe molecule relative to the nanoantenna in the field mapping experiment. 
antenna excited close to the dipolar resonance (Figure 1a). When exciting from the far-field with a wave propagating in the $\mathrm{z}$-direction and polarized along the long axis of the nanoantenna ( $\mathrm{x}$ direction), it is the x-polarized component of the induced near-field that will interfere with the excitation wave. Both the near-and far-field distributions of the nanoantenna are altered according to Eq. 1. Any near-field component induced in the other dimensions $(y, z)$ will not experience interference as the exciting wave has no field component in these directions with which to interfere. This underscores the vectorial nature of this effect. The far-field interference pattern, away from the nanoantenna, is clearly observed in the numerically calculated x-field component contribution to the intensity map in the xz-plane, see Figure $1 \mathrm{~b}$. This interference occurs between the two propagating fields: the scattered far-field of the nanoantenna and the incident plane excitation wave. This generates a classic fringe pattern with a period of $\lambda / 2$ (Figure $1 b$ ). Here, the phase difference is acquired mainly due to the counter propagation of two fields in space and the resonance of the nanoantenna. Close to the nanoantenna, in the near-field (see dashed line Figure 1b), the nanostructured amplitude and phase distributions are responsible for a local interference effect between the localized scattered near-field and the propagating incident field. In the centre of the hotspots of the nanoantenna, any interference is negligible due to the mismatch of amplitude between the two fields; the scattered field amplitude here far exceeds the incident field amplitude. However, when the strength of the near-field components of the nanoantenna are comparable in amplitude to the incident field, which is true for positions slightly away from the hotspot, this interference becomes crucial to the observed intensity pattern. Crucially, at some nanometers below (or away from) the nanoantenna the fields have significant areas of similar amplitude and so the interference effect dominates. To fully understand the implications of this near-field interference we use a molecular probe positioned below the nanoantenna at the nanometric distance for interference to map the intensity in the xy-plane. 


\section{Local interference probed by single molecules}

Experimental observation of such local effects remains a challenge. To be observable, a nano-sized detector needs to be placed at the position of the interference to record the resultant field intensity. Due to the rapidly decaying near-field of the nanoantenna, the detector must be placed within the decay length of the field. Here, we choose nanometer sized single fluorescent molecules as local nanoprobes, with a fixed absorption dipole moment p. We use terrylene diimide (TDI) dye molecules as near-field probes in our measurements. A single molecule sample is prepared by spin-coating a dilute $\left(10^{-9} \mathrm{M}\right)$ solution of terrylene diimide (TDI) dye molecules in $1 \%$ PMMA [poly(methyl methacrylate)] onto a cleaned glass coverslip. This provides a thin $(\approx 20 \mathrm{~nm})$ layer of fluorescent molecules in a PMMA matrix. The emission intensity of the molecule is recorded by scanning a nanorod shaped resonant aluminum $(\mathrm{Al})$ dipole nanoantenna (length $=170 \mathrm{~nm}$, width $=$ $50 \mathrm{~nm}$, and height $=50 \mathrm{~nm})$ over $(\approx 10-15 \mathrm{~nm}$ above $)$ a single molecule excited below saturation and held within the focus of an excitation field. The nanoantenna is scanned in steps of $5 \mathrm{~nm}$ using the experimental setup developed by us and described elsewhere. ${ }^{13}$ The nanoantenna is fabricated at the apex of a glass fiber tip with its long axis parallel to $\mathrm{x}$, and excited by circularly polarized light at $633 \mathrm{~nm} \cdot{ }^{21,22}$

A molecule (p) placed at any position $\mathbf{r}$ close to the nanoantenna experiences the total field $\mathbf{E}_{t o t}$ (Figure 1) and thus directly probes the interference. The resultant fluorescence signal is expressed as:

$$
I_{f} \propto\left|\mathbf{p} . \mathbf{E}_{t o t}(r)\right|^{2}=\sum_{i}\left|p_{i} \cdot E_{t o t}^{i}\right|^{2}
$$

Selecting a specific orientation of the absorption dipole moment of the molecule allows us to probe a specific (or combination of) field component(s). ${ }^{23,24}$ We define the orientation of a molecule in the xyz-plane using conventional spherical coordinates with a polar angle $(\theta)$ away from the z-axis and azimuthal angle $(\phi)$ away from the $x$-axis in the $x-y$ plane. On probing the $x$-field component with a molecule orientated in the xy-plane and along the x-axis, we obtain an intensity pattern (Figure 2a) 
with two hotspots surrounded by a dramatic low-field intensity plateau before recovering intensity again further out, much like a canyon. The resultant intensity has been modulated by the local interference due to sub-wavelength changes in both amplitude and phase distributions. 


\section{Total intensity maps (with interference)}

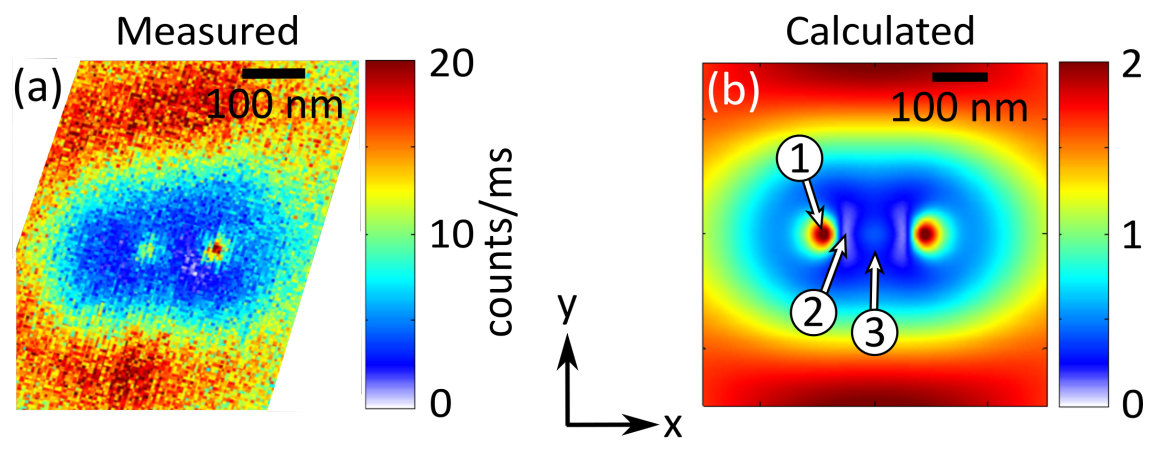

\section{Scattered contribution only (no interference)}

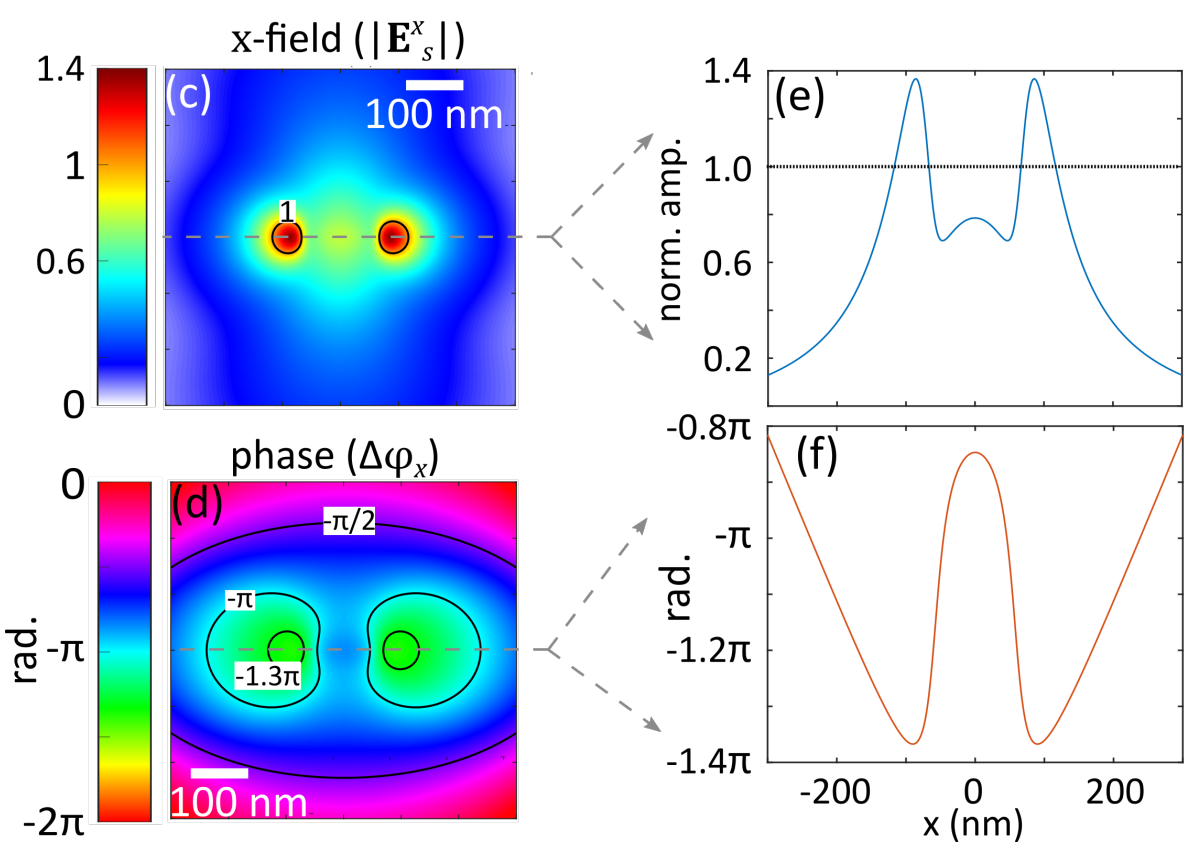

Figure 2: Effect of the amplitude and phase distributions on the local interference. (a) The measured $\mathrm{x}$-field intensity map of a resonant Al nanoantenna using a single fluorescent molecule shows a typical two hotspots pattern surrounded by a low-field intensity plateau. (b) The calculated x-field intensity of the resonant nanoantenna (see Figure 1) in the presence of the incoming field $\left(\mathbf{E}_{i n}\right)$, showing similar pattern as in the measured map. The maps clearly show the change in the field strength of the nanoantenna due to local interference. The destructive interference reduces the intensity in the middle (position 3), while the intensity becomes minimum at position 2 . The hotspots are situated within a low-field plateau region with intensity less than the incident light $I_{\text {in }}<$ 1; blue colors on plot). Calculated (c) amplitude (d) phase maps of the scattered x-field component of the same nanoantenna, considering the scattered field $\left(\mathbf{E}_{s}\right)$ component alone. Isolines of field amplitude with a value 1 is indicated in (c). Similarly, isolines of phase $-\pi / 2,-\pi$, and phase around the hotspots are shown in (d). Line profiles of the (e) field amplitude and (f) phase along the long axis of nanoantenna through the hotspots (see the dashed line), showing the variation of amplitude and phase along the nanoantenna. This is responsible for the change in the resultant intensities at positions 1, 2, and 3 in (b) due to interference with the incoming field. For the calculated maps, field is calculated in the plane $15 \mathrm{~nm}$ below the nanoantenna and is normalized to the excitation field. 
Figure $2 \mathrm{~b}$ shows the resulting intensity $\left(I_{\text {tot }}^{x}\right)$ from finite-difference time domain (FDTD) calculations mimicking the experimental conditions including the local interference effect between the scattered and incident fields. The intensity values are normalized to the intensity of the excitation field $\left(I_{\text {in }}=1\right)$. The calculated pattern matches well with the experimental measurements in Figure 2a and provides further clarity on the key features of the local interference effect present here. The intensity is enhanced at the two strong sub-wavelength hotspots (position 1) as is characteristic of the first-order resonance mode of the nanoantenna. ${ }^{21,25}$ Between the hotspots the intensity decreases (position 3), and reaches a minimum value at position 2 (white region), which is not resolved in the experimental case. This is in stark contrast to the expected intensity map if local interferences are not accounted for. To understand the origin of the local interference that creates the pattern observed in Figure 2a, b, we crucially need to understand the amplitude and phase map of the local field.

In Figure 2c, we present the FDTD calculated field amplitude $\left(\left|\mathbf{E}_{s}^{x}\right|\right)$ distribution of the x component of the scattered near-field matching the experimental conditions but with local interference effects removed, see Figure 2c. In this hypothetical situation, we see two strong sub-wavelength hotspots similar to the experimental case, however, the field here simple decays as we move away from the hotspots. The low-field plateau feature with later field recovery surrounding the hotspots is not observed. Perhaps most striking is the presence of what looks like a third hotspot (of lower maximum amplitude) in between the two principle hotspots. In fact, since this supposed hotspot is of low field amplitude (close to and below that of the incident field amplitude) it is very susceptible to the local interference effect and when this is taken into account becomes the minimum we observe in the experimental case.

Figure $2 \mathrm{~d}$ plots the spatial phase of the field around the nanoantenna relative to the excitation field at the position where the field is monitored. The phase map shows a similar two-lobed pattern (albeit less confined) with an almost $\pi / 2$ shift between the position of the hotspot and the middle of the nanoantenna. According to Eq. 1, the nanostructuring of the phase permits the field to locally interfere, constructively or destructively, relative to the constant phase of the incoming field. The spatial phase map here should not be confused with the $\pi$ temporal phase difference between the two 
hotspots in dipolar nanoantennas. The temporal phase shift is a function of the incident radiation frequency, which is the same as the scattered frequency and thus any effect averages out over an optical cycle and is therefore not needed to understand this spatial phase induced intensity map. The origin of the low-field plateau, field recovery and the suppressed central hotspot can now be observed as the phase makes a $\pi / 2$ shift at the border of the large hotspots both right in the centre and the surrounding area. This measurement confirms the presence of the local interference effect and its influence on the near-field distribution of the resonant nanoantenna.

Furthermore, the area surrounding the hotspots has phase between $-2 \pi$ and $-\pi / 2$ relative to the incident field (see isoline $-\pi / 2$ in Figure $2 \mathrm{~d}$ ). This causes destructive interference at all those positions including the hotspots in accordance to Eq. 1 and creates the low-field plateau in Figure 2a,b. However, the destructive interference has a small effect on the hotspots due to the field localization and enhancement. The line profiles (dashed line in Figure 2c,d) through the hotspots in the field and phase map clearly demonstrate this behavior in Figure 2e,f. At and around hotspots where the field amplitude is $>1$ (points above unity amplitude in Figure 2e), the resultant field intensity remains enhanced (more than the incident field intensity, point 1 Figure $2 b$ ). All the other positions see reduction in the their resultant intensities as seen at point 2 and 3 in Figure 2 b. The point 2 is a near perfect destructive interference with intensity reaching zero caused by a close to $-\pi$ phase relative to the incident field, see Figure $2 \mathrm{f}$.

\section{Effect of incident polarization on the local interference}

According to Eq. 1, different field components contribute to the local interference due to the 3D vectorial nature of the near-field, depending on the polarization state of the incoming field. A circular polarized wave is composed of $\mathrm{x}$ - and $\mathrm{y}$-field components with equal amplitude and $\pi / 2$ phase difference. On exciting the dipole nanoantenna, such an excitation field predominantly excites the dipolar mode while the interaction of the nanoantenna with the y-field remains weak. Nevertheless, considering Eq. 1, both the $\mathrm{x}$ - and $\mathrm{y}$-field components of the nanoantenna can be 
affected by the local interference effect. A molecule in close vicinity to the nanoantenna experiences this combination of fields, which strongly depends on the orientation of the molecule in space.

Figure 3a-e show measured intensity maps of the nanoantenna, excited with circularly polarized light and probed by molecules of different orientations. Depending on the molecule orientation, the maps exhibit strong variations in their intensity patterns showing different fluorescence intensities away from the nanoantenna hotspots, symmetric or asymmetric hotspots, bright and dark spots as well as butterfly shaped distributions. In many cases the intensity pattern is substantially different from that expected considering nanoantenna scattering alone, and we must use the local interference effect to fully understand the observations. Due to the circular polarization of the incoming field, molecules oriented along the xy-plane will be the most affected by the local interference effect. On the other hand, molecules oriented principally along the z-axis will be less sensitive to the interference. Apart from the polarization of the excitation field, which is responsible for the local interference, we also need to take into account the orientation of the single molecules with respect to the nanoantenna to interpret the intensity maps as illustrated by Eq. 2 .

To illustrate the effect of orientation we take an out-of-plane molecule oriented in the z-direction $\left(\theta=0^{\circ}, \phi\right.$ undefined $)$ to probe our nanoantenna electric field component in the z-direction. Experimentally we find such molecule as, without a nanoantenna, in the microscope focus we cannot directly excite it, so it appears dark unless a nanoantenna is present. Figure 3a shows the measured intensity map for such a molecule, Figure $3 f$ shows the matching FDTD calculated map (with interference) and Figure $3 \mathrm{k}$ the hypothetical case considering only the scattering component to the intensity (no interference). In Figure 3a we see the characteristic double hotspot pattern of a dipolar $(\lambda / 2)$ nanoantenna. From the calculations we can see that this pattern remains unchanged for both calculations, i.e. there is no local interference effect for the z-orientated molecule. This experimental result clearly supports what we expect from before, as the incident field has zero component in the z-direction to interfere with the z-component of the scattered field. 
Measured
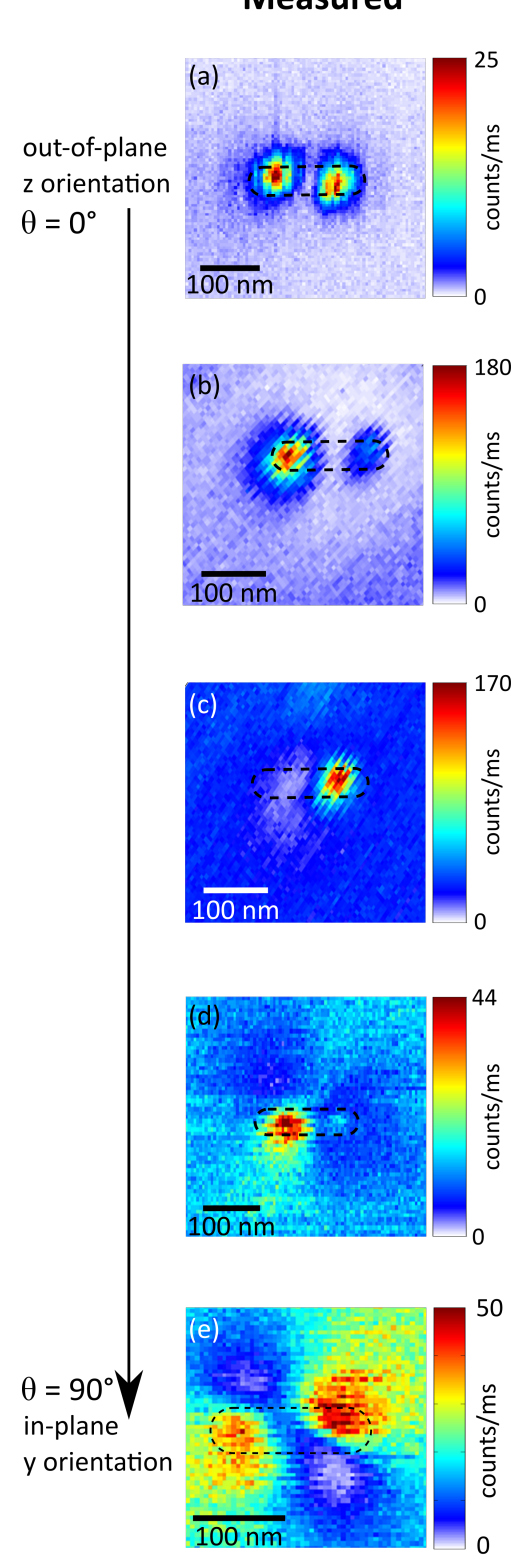
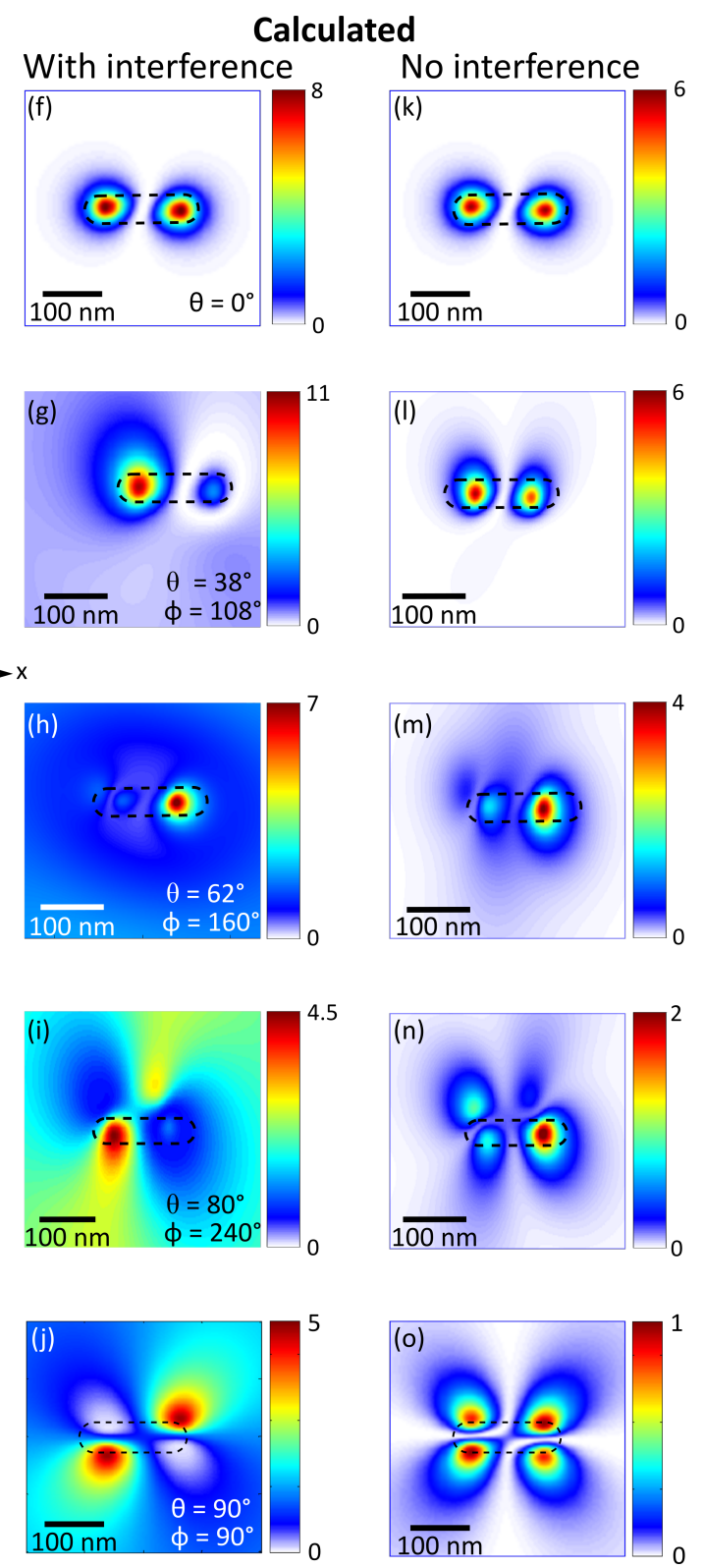

Figure 3: (a-e) Measured intensity maps of the nanoantenna excited by circularly polarized light at $633 \mathrm{~nm}$ and probed by five single molecules of different orientations. The variation and asymmetry in the intensity patterns arise due to the local interference of $\mathbf{E}_{\text {in }}$ and $\mathbf{E}_{s}$, and the projection of the resultant field on the molecule's dipole moment. (f-j) The finite-difference time-domain (FDTD) calculations of the intensity patterns at $15 \mathrm{~nm}$ below the nanoantenna when excited by circular polarized light to match the experiments. The calculations are performed considering the full field (with the local interference effect), and using molecule orientation defined in the figure by $\theta$ and $\phi$. The calculated intensity maps match very well with the measured maps, showing values of $\theta$ and $\phi$ closely describing the original orientation of the molecules. (k-o) Shows calculated maps with the effect of interference removed, i.e. intensity based on the scattering electric field amplitude $\mathbf{E}_{S}$ only. This shows striking differences. The asymmetry shown here is due to the orientation of the molecules. All scale bars are linear. 
On the opposite extreme are probe molecules oriented in the plane of the nanoantenna, which is also the plane of polarization of the incident light $\left(\theta=90^{\circ}\right)$. In Figure 2 we saw the effect on such a molecule aligned along the long axis $\left(\mathrm{x} ; \phi=0^{\circ}\right)$ of the nanoantenna to probe the $\mathrm{x}$-component of the electric field. In Figure 3e we probe the y-component of the electric field using a molecule aligned along the nanoantenna short-axis $\left(\mathrm{y} ; \phi=90^{\circ}\right)$ along with its FDTD calculation in Figure 3j. Remarkably the pattern shows two bright hotspots diagonally opposite and two dark hotspots on the opposite diagonal, instead of the expected four-lobed bright hotspot pattern (Figure 3o) for such a molecule when local interference is neglected. Here the powerful effect of constructive and destructive interference is visible on the hotspots themselves effectively creating nanoscale enhanced and suppressed areas for the excitation of the molecule.

In between the extreme cases of molecules orientated in-plane along the $\mathrm{x}$ or $\mathrm{y}$ axis or outof-plane aligned along $\mathrm{z}$, there exists infinite possible orientations of the molecule. These allow us to start to see the practical implications of local interference and how we can tailor its shape based on the molecular absorption dipole moment orientation. By using different probe molecule orientations we access different local interference states which are a combination of the different electric field amplitude and phase distributions along the Cartesian axis. Figure $3 b, c, d$ shows three such molecules increasingly orientated in-plane (increasing $\theta$ ) and with differing $\phi$ orientation. By comparing to the calculated intensity maps Figure 3e,g,i (with interference) and Figure 31,m,n (without interference) it is clear to see the different extent to which local interference plays a role, especially as the orientation becomes more in-plane. We also observe that this effect can produce rather strange intensity patterns which at first glance seem hard to interpret based on a dipole nanoantenna. In fact, using the local interference, the exact orientations of these molecules were deduced by matching the calculated patterns of different molecular orientations to the experimental data.

In addition to the modification to the field distribution, it is clear that significant enhancement (or indeed suppression) of the near-field intensities can be produced by this local near-field interference. In these maps, we see how the combination of local interferences involving the two in-plane 
components drastically alters the resultant intensity patterns.

\section{Local interference for hotspot switching}

Instead of changing the molecule orientation on the nanoscale, these patterns can also be altered by changing the excitation conditions in the macroworld, going from linear to circular polarization excitation, without need for the nanomanipulation previously demonstrated. This opens up the powerful possibility of switching the local electric field pattern by leaving the nanoenvironment unchanged and simply altering the far-field illumination conditions.

For the dipole nanoantenna, the local interference effect creates an asymmetric four-lobed pattern for the $y$-field component instead of the expected symmetrical four-lobed pattern, as we have seen above (Figure 3j,o). Here, in Figure 4, we further explore this interesting four-lobed pattern by looking at the phase map and the effect of the incident polarization. Indeed, Figure 4d,e shows two-bright spots along one diagonal (point 1 and 3) and two-dark spots with almost no field on the opposite diagonal (point 2 and 4). In the hypothetical intensity map without interference (Figure $4 b$ ), the four lobes of the $y$-field component of the nanoantenna are bright with almost equal intensity. Each lobe, or 'hotspot', has a phase difference of $\pi$ compared to its neighbors (Figure 4a). Thus, the amplitude and phase relations of the incoming y-field is such that it results in destructive interference for the two-dark spots while consequently the two-bright spots present constructive interference with 5-fold intensity enhancement (Figure 4d). The position of the bright and dark spots have their origin in the phase distribution, notably the phase difference between the lobes. Thus, by changing the polarization state of the incoming field from left to right circular polarization, we can invert the position of the bright and dark hotspots (Figure $4 \mathrm{~d}, \mathrm{e}$ ). This is since the phase difference between the $\mathrm{x}$ - and $\mathrm{y}$-field components of the incoming field is $\pi$-shifted, passing from $\pi / 2$ to $-\pi / 2$ (Figure $4 c$ ) interchanging the constructive and destructive local interference conditions. This way, external polarization switching of the nanosized hotspots can be realized directly using the near-field interference effect. 

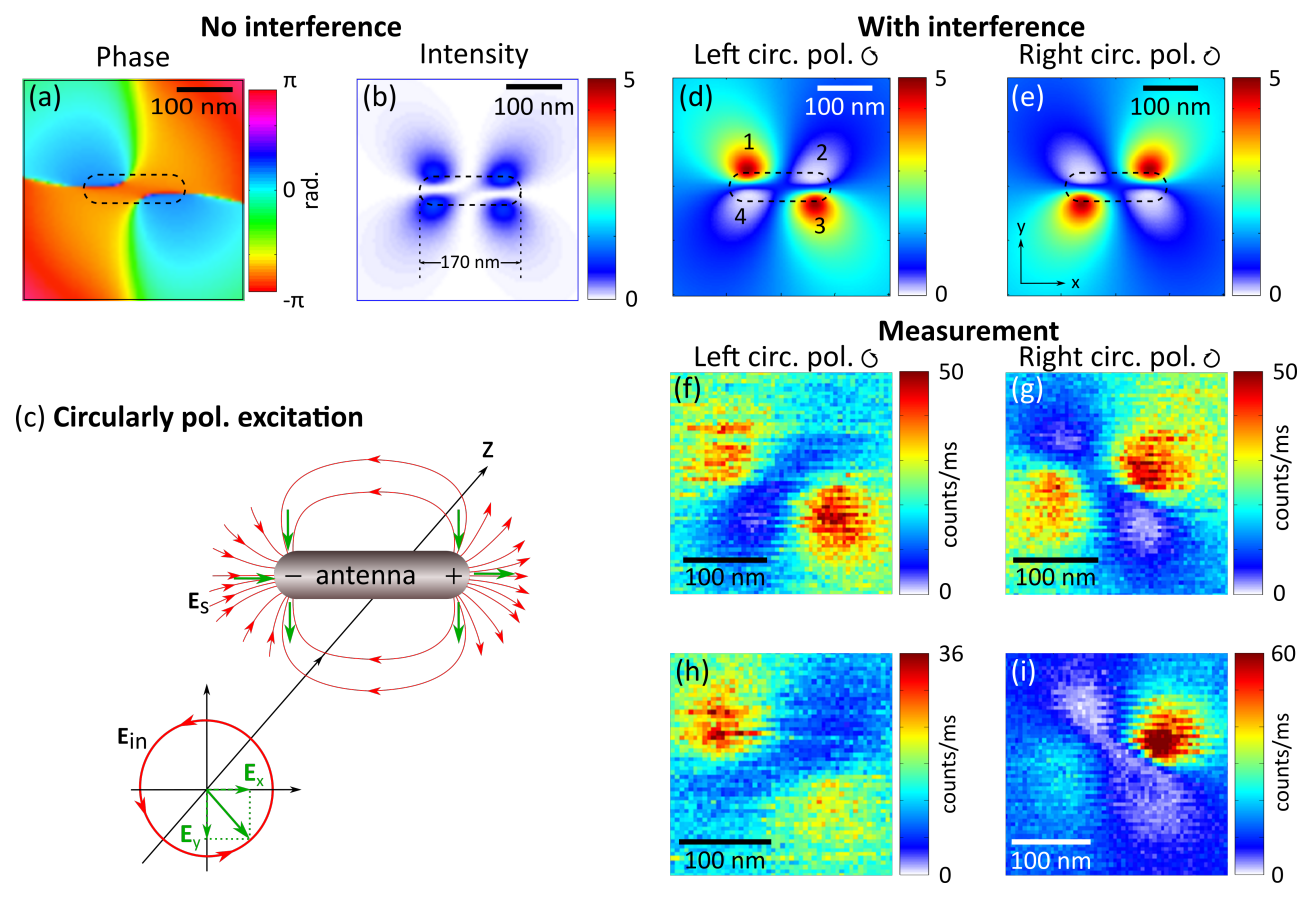

Figure 4: Switching hotspots. FDTD calculated (a) phase and (b) intensity maps of the y-field component of the nanoantenna without local interference when excited by circularly polarized light. (c) A schematic of a dipole nanoantenna and its field lines in the xy-plane when excited by circularly polarized light propagating along the z-direction. Different colors are used in the figure to indicate the field components of the incident and scattered light. The FDTD simulated $y$-field maps of the dipole nanoantenna for two different excitation polarizations conditions: (d) left circularly polarized light and (e) right circularly polarized light. In (d) the alternating bright/dark hotspot diagonal lobe pattern caused by local interference is apparent. The field intensity completely vanishes at positions 2 and 4 due to destructive interference. The constructive interference also further enhances the field by a factor of 5 at the hotspots, positions 1 and 3. In (e) the hotspot positions are flipped by $90^{\circ}$. A dashed outline represents the nanoantenna. (f-i) Measured maps for the two polarization states using y-oriented single molecules. All scale bars are linear.

We experimentally demonstrate this switching effect by using y-oriented molecules as local nanoprobes (Figure 4f-i). All the measured maps clearly show two hotspots, which switch with high contrast on changing the incoming polarization state from left to right circularly polarized. The two maps from two different molecules (of same orientation) for each polarization state emphasize the reproducibility of the images and match very well with the calculated maps (Figure 4d,e). A small asymmetry in some maps, mainly in Figure $4 \mathrm{i}$ can be attributed to the orientation of the molecule not perfectly aligned in the y-direction. Thus, the external control of the polarization state of the incident wave allows the shaping of the near-field distribution of the dipole nanoantenna and 
dramatic hotspot switching.

According to Eq. 2, depending on the incoming polarization state, the local interference can modulate the total intensity map, where total intensity refers to the intensity from the combined field components in $\mathrm{x}, \mathrm{y}$ and $\mathrm{z}$. Simulations and experiments confirm this modification, revealing a small but notable effect as shown in Figure 5. For experimental demonstration, we now use fluorescent nanobeads, composed of many fluorescent molecules of all orientations, as probes instead of single molecular dipoles to ensure sensitivity to all field components. Going from left to right circularly polarized excitation one observes a small rotation of the hotspot axis in the clockwise

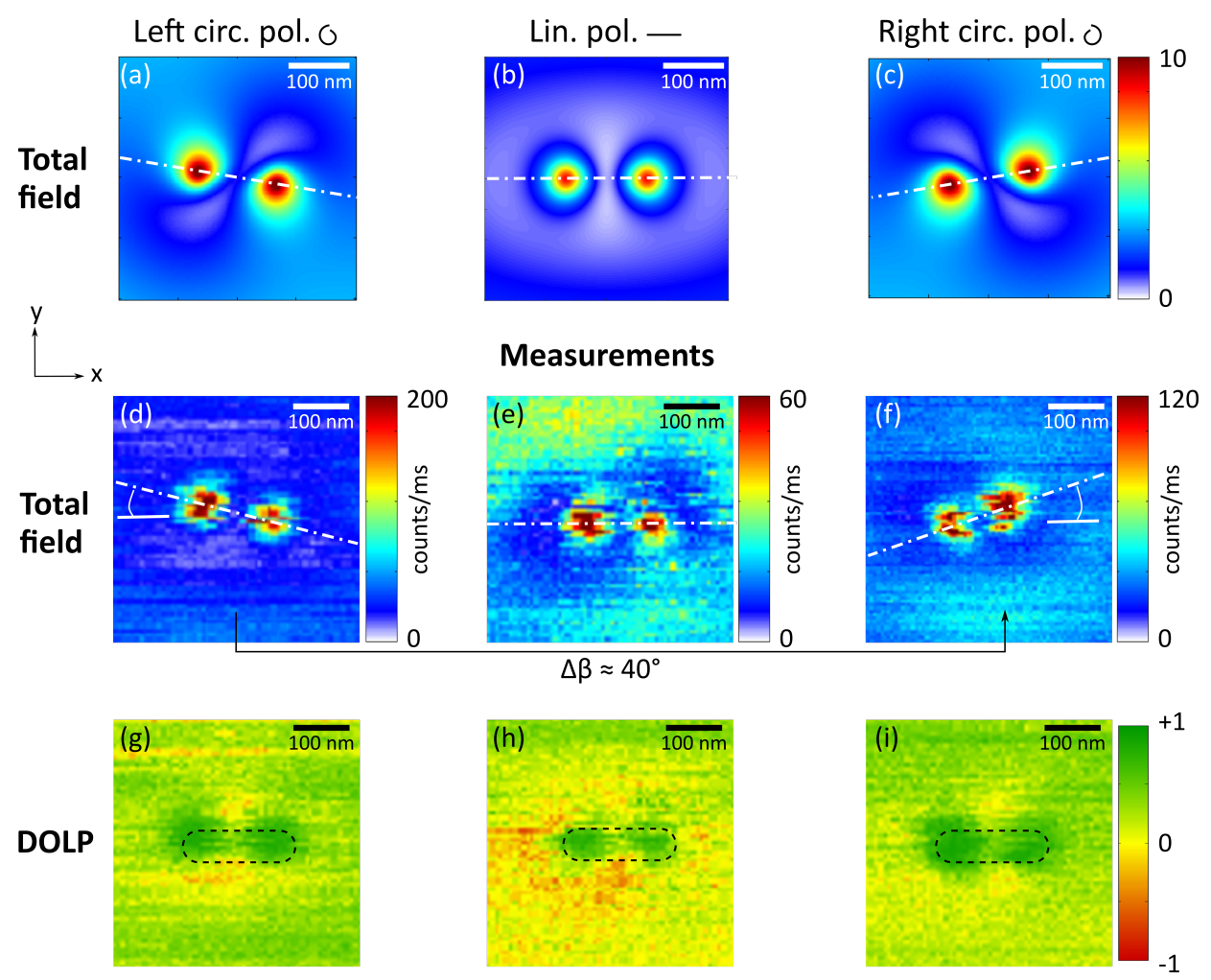

Figure 5: Local interference effect on the total field. The calculated total electric field maps of the nanoantenna for three different excitation polarizations states. (a) Left circularly, (b) linear, (c) right circularly polarized. The two hotspots can be seen rotated away from the nanoantenna axis (in opposite directions) in the case of left and right circularly polarization, while the hotspots follow the horizontal line of the nanoantenna for linear polarization (see the dashed-dotted line). (d-f) The measured total field intensity maps show the same effect as the calculations for the different polarization states. We measure the total rotation angle $\Delta \beta \approx 40^{\circ}$ between the two cases (d,f). (g-i) DOLP maps, exhibiting emission polarization, confirm the main axis of the nanoantenna along the $\mathrm{x}$ (horizontal) axis in all the three cases. All scale bars are linear. 
and anticlockwise directions, respectively (Figure 5d,f), while for the linearly polarized state, the two hotspots lie on a horizontal line in Figure 5e. The experimental results match very well with the calculated maps.

To verify that the hotspot rotation is not an artifact due to changes in nanoantenna orientation, we determine the degree of linear polarization (DOLP). The DOLP is an intensity (excitation) free parameter defined as DOLP $=\left(I_{x}-I_{y}\right) /\left(I_{x}+I_{y}\right)$, where $I_{x}$ and $I_{y}$ are the collected fluorescence intensities of $\mathrm{x}$ and $\mathrm{y}$ polarized light respectively. The DOLP measures the change in the emission

polarization of the nanobeads when coupling efficiently with the nanoantenna. ${ }^{13,26}$ All hotspot positions show a linear x-polarized emission (green spots in Figure 5g-i) and are thus free from any interference effects and reveal the true orientation of the nanoantenna to be along the horizontal axis, consistent in all three measurements. This confirms that the rotation of the hotspot positions around the nanoantenna axis is driven by a change in the polarization state of the excitation light. Externally setting the polarization state of the incoming field allows us to shape the near-field electric field and control the excitation hotspot positions. It's important to mention that the local near-field interference is a pure excitation based phenomena and doesn't affect the emission of a coupled single emitter as interference is observed only at excitation wavelength and the local density of optical states available for emission is unaffected by the choice of excitation conditions. Although, it is worth noting that the emission enhancement may obscure the local interference if the nanoantenna is resonant with the emission wavelength, which is not the case here. ${ }^{26-28}$

\section{Conclusion and outlook}

We have studied in detail the effect of local near-field interference in the near-field of an optical nanoantenna and shown how enhancement and depletion of the local field can be manipulated from the far-field by changes in the polarization of the excitation beam. We have experimentally demonstrated this local interference effect on a resonant Al dipole nanoantenna using single fluorescent molecules as local dipole field probes. A significant change in the measured field 
distribution was observed using differently oriented molecules. The in-plane nanoantenna field components were modified the most by the interference due to the in-plane incident polarization, while the out-of-plane field component remained largely unaffected . We exploited the local interference effect to exert polarization control of the hotspots of a nanoantenna. This polarizationcontrolled switching can be applied to switch or control the optical near-field in an all optical nanocircuitry or to switch illumination sources between different quantum emitters in compact single photon sources.

\section{Acknowledgement}

N.F.v.H. acknowledges the financial support by the European Commission through ERC Advanced Grant 670949-LightNet. Authors acknowledge support by the Ministry of Science, Innovation \& Universities (PGC2018-096875-B-I00), the Ministry of Economy (FIS2015-69258-P and "Severo Ochoa" program for Centers of Excellence in R\&D SEV-2015-0522), the Catalan AGAUR (2017SGR1369), Fundació Privada Cellex, Fundació Privada Mir-Puig, and Generalitat de Catalunya through the CERCA program.

\section{Methods}

\section{Nanoantenna fabrication}

We use a nanorod shaped antenna which is designed for the first-order resonance mode i.e. a dipole pattern at $633 \mathrm{~nm}$. The nanoantenna is fabricated at the apex of a tapered optical fiber, which provides support to attach the nanoantenna to the piezo scanner head of the scanning near-field microscope. The nanoantenna is fabricated on a $50 \mathrm{~nm}$ thick layer of evaporated aluminum, sculpted into the correct geometry using focused-ion-beam (FIB) milling, forming a nanorod shape with a length of $170 \mathrm{~nm}$ and width and depth of $50 \mathrm{~nm}$ (see Figure 6a,b). 


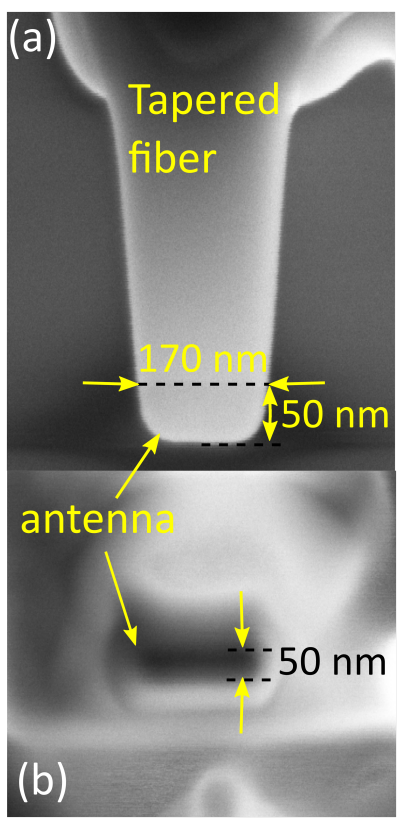

Figure 6: Scanning electron microscope image of FIB fabricated nanoantenna on the apex of a tapered fiber. (a) Side view and (b) Top view.

\section{Measurement setup}

All experiments were performed in a home-built scanning near-field microscope which is based on an inverted confocal microscope with piezo-electric scanners capable of scanning both sample and nanoantenna. A shear-force feedback mechanism is used to control nanoantenna- sample distance. The nanoantenna is positioned in the focus of diffraction-limited linearly/circularly polarized excitation laser beam $(\lambda=633 \mathrm{~nm})$ using a 100x 1.3 NA oil-immersion microscope objective and is scanned with nanometer accuracy in the close vicinity $(\approx 10-15 \mathrm{~nm})$ of a fluorescent molecule to acquire near-field intensity maps of the nanoantenna. In the emission path, a dichroic beam splitter and dielectric long pass fluorescence filter are used to separate the excitation light from the molecule fluorescence. The fluorescence is confocally imaged onto two fast avalanche photodiodes (APD) after passing through polarizing beam splitter to enable polarization resolved imaging. 


\section{References}

(1) Novotny, L.; van Hulst, N. Antennas for light. Nature Photonics 2011, 5, 83-90.

(2) Biagioni, P.; Huang, J.-S.; Hecht, B. Nanoantennas for visible and infrared radiation. Reports on Progress in Physics 2012, 75, 024402.

(3) Schuller, J. A.; Barnard, E. S.; Cai, W.; Jun, Y. C.; White, J. S.; Brongersma, M. L. Plasmonics for extreme light concentration and manipulation. Nature Materials 2010, 9, 193-204.

(4) Kawata, S.; Inouye, Y.; Verma, P. Plasmonics for near-field nano-imaging and superlensing. Nature Photonics 2009, 3, 388-394.

(5) Kneipp, K.; Wang, Y.; Kneipp, H.; Perelman, L.; Itzkan, I.; Dasari, R.; Feld, M. Single Molecule Detection Using Surface-Enhanced Raman Scattering (SERS). Physical Review Letters 1997, 78, 1667-1670.

(6) Zentgraf, T.; Dorfmüller, J.; Rockstuhl, C.; Etrich, C.; Vogelgesang, R.; Kern, K.; Pertsch, T.; Lederer, F.; Giessen, H. Amplitude- and phase-resolved optical near fields of split-ringresonator-based metamaterials. Optics Letters 2008, 33, 848.

(7) Schnell, M.; Garcia-Etxarri, A.; Huber, A. J.; Crozier, K. B.; Borisov, A.; Aizpurua, J.; Hillenbrand, R. Amplitude- and Phase-Resolved Near-Field Mapping of Infrared Antenna Modes by Transmission-Mode Scattering-Type Near-Field Microscopy. Journal of Physical Chemistry C 2010, 114, 7341-7345.

(8) Dorfmüller, J.; Vogelgesang, R.; Khunsin, W.; Rockstuhl, C.; Etrich, C.; Kern, K. Plasmonic nanowire antennas: experiment, simulation, and theory. Nano Letters 2010, 10, 3596-3603.

(9) Deutsch, B.; Hillenbrand, R.; Novotny, L. Near-field amplitude and phase recovery using phase-shifting interferometry. Optics Express 2008, 16, 494-501. 
(10) Balistreri, M. L.; Korterik, J. P.; Kuipers, L.; van Hulst, N. F. Local observations of phase singularities in optical fields in waveguide structures. Physical Review Letters 2000, 85, 294-297.

(11) Schnell, M.; Garcia-Etxarri, A.; Alkorta, J.; Aizpurua, J.; Hillenbrand, R. Phase-resolved mapping of the near-field vector and polarization state in nanoscale antenna gaps. Nano Letters 2010, 10, 3524-3528.

(12) Yu, N.; Genevet, P.; Kats, M. a.; Aieta, F.; Tetienne, J.-P.; Capasso, F.; Gaburro, Z. Light Propagation with Phase Discontinuities Reflection and Refraction. Science 2011, 334, 333337.

(13) Singh, A.; Calbris, G.; van Hulst, N. F. Vectorial Nanoscale Mapping of Optical Antenna Fields by Single Molecule Dipoles. Nano Letters 2014, 14, 4715-4723.

(14) De Angelis, L.; Alpeggiani, F.; Di Falco, A.; Kuipers, L. Persistence and Lifelong Fidelity of Phase Singularities in Optical Random Waves. Physical Review Letters 2017, 119, 203903.

(15) Berkhout, A.; Koenderink, A. F. Perfect Absorption and Phase Singularities in Plasmon Antenna Array Etalons. ACS Photonics 2019, 6, 2917-2925.

(16) Lin, J.; Mueller, J. P. B.; Wang, Q.; Yuan, G.; Antoniou, N.; Yuan, X.-C.; Capasso, F. Polarization-Controlled Tunable Directional Coupling of Surface Plasmon Polaritons. Science 2013, 340, 331-334.

(17) Rodríguez-Fortuño, F. J.; Marino, G.; Ginzburg, P.; O’Connor, D.; Martínez, A.; Wurtz, G. A.; Zayats, A. V. Near-field interference for the unidirectional excitation of electromagnetic guided modes. Science 2013, 340, 328-330.

(18) le Feber, B.; Rotenberg, N.; Kuipers, L. Nanophotonic control of circular dipole emission. Nature Communications 2015, 6, 6695.

(19) Rotenberg, N.; Kuipers, L. Mapping nanoscale light fields. Nature Photonics 2014, 8, 919-926. 
(20) le Feber, B.; Sipe, J. E.; Wulf, M.; Kuipers, L.; Rotenberg, N. A full vectorial mapping of nanophotonic light fields. Light: Science and Applications 2019, 8, 28.

(21) Singh, A.; Hugall, J. T.; Calbris, G.; van Hulst, N. F. Fiber-based Optical Nanoantennas for Single Molecule Imaging and Sensing. Journal of Lightwave Technology 2015, 33, 2371-2377.

(22) Neumann, L.; van 't Oever, J.; van Hulst, N. F. A resonant scanning dipole-antenna probe for enhanced nanoscale imaging. Nano letters 2013, 13, 5070-5074.

(23) Betzig, E.; Chichester, R. J. Single molecules observed by near-field scanning optical microscopy. Science 1993, 262, 1422-1425.

(24) Novotny, L.; Beversluis, M.; Youngworth, K.; Brown, T. Longitudinal Field Modes Probed by Single Molecules. Physical Review Letters 2001, 86, 5251.

(25) Ghenuche, P.; Cherukulappurath, S.; Taminiau, T. H.; van Hulst, N. F.; Quidant, R. Spectroscopic Mode Mapping of Resonant Plasmon Nanoantennas. Physical Review Letters 2008, $101,116805$.

(26) Taminiau, T. H.; Stefani, F. D.; Segerink, F. B.; van Hulst, N. F. Optical antennas direct single-molecule emission. Nature Photonics 2008, 2, 234-237.

(27) Singh, A.; De Roque, P. M.; Calbris, G.; Hugall, J. T.; Van Hulst, N. F. Nanoscale Mapping and Control of Antenna-Coupling Strength for Bright Single Photon Sources. Nano Letters 2018, 18, 2538-2544.

(28) Anger, P.; Bharadwaj, P.; Novotny, L. Enhancement and Quenching of Single-Molecule Fluorescence. Physical Review Letters 2006, 96, 113002. 